\title{
Singing Exercises Improve Sleepiness and Frequency of Snoring among Snorers-A Randomised Controlled Trial ${ }^{*}$
}

\author{
M. P. Hilton ${ }^{1}$, J. Savage ${ }^{1}$, B. Hunter ${ }^{1}$, S. McDonald ${ }^{1}$, C. Repanos ${ }^{1}$, R. Powell ${ }^{2}$ \\ ${ }^{1}$ Department of Otolaryngology, Head \& Neck Surgery, Royal Devon \& Exeter NHS Foundation Trust, Exeter, UK \\ ${ }^{2}$ Research \& Development Directorate, Royal Devon \& Exeter NHS Foundation Trust, Exeter, UK \\ Email: malcolmhilton@nhs.net
}

Received December 20, 2012; revised March 2, 2013; accepted April 15, 2013

Copyright (C) 2013 M. P. Hilton et al. This is an open access article distributed under the Creative Commons Attribution License, which permits unrestricted use, distribution, and reproduction in any medium, provided the original work is properly cited.

\begin{abstract}
Objectives: To assess the effectiveness of regular singing exercises in reducing symptoms of snoring and sleep apnoea. Methods: A prospective single blinded randomised controlled trial was conducted in the otolaryngology department of a UK teaching hospital (Exeter). 127 adult patients with a history of simple snoring or sleep apnoea were recruited. 93 patients completed the study. Patients were excluded because of severe sleep apnoea (apnoea index $>40$ ), or morbid obesity (BMI > 40). The study group completed a self-guided treatment programme of singing exercises contained on a 3CD box set, performed for 20 minutes daily. Outcome measures included the Epworth Sleepiness Scale, the SF-36 generic quality of life assessment tool, visual analogue scales (VAS range 0 - 10) of snoring loudness and frequency, and visual analogue scale of compliance (for intervention group). Results: The Epworth scale improved significantly in the experimental group compared to the control group (difference -2.5 units; $95 \% \mathrm{CI}-3.8$ to $-1.1 ; \mathrm{p}=0.000$ ). Frequency of snoring reduced significantly in the experimental group (difference $-1.5 ; 95 \% \mathrm{CI}-2.6$ to $-0.4 ; \mathrm{p}=0.01$ ), and loudness of snoring showed a trend to improvement which was non-significant (difference $-0.8 ; 95 \% \mathrm{CI}-1.7$ to $0.1 ; \mathrm{p}$ $=0.08$ ). Compliance with exercises was good; median 6.6 (quartiles $=4.1,8.3$ ). Conclusions: Improving the tone and strength of pharyngeal muscles with a 3 months programme of daily singing exercises reduces the severity, frequency and loudness of snoring, and improves symptoms of mild to moderate sleep apnoea.
\end{abstract}

Keywords: Snoring; Obstructive Sleep Apnoea; Upper Airway Resistance; Pharyngeal Exercises

\section{Introduction}

Upper airway resistance during sleep can present with a range of symptoms from simple snoring (SS) through to severe obstructive sleep apnoea (OSA). The prevalence of OSA is $2 \%-4 \%$ in men, and $1 \%-2 \%$ in females. Severe sleep apnoea has a prevalence of $0.3 \%-0.7 \%$ [1]. The prevalence of SS is much higher [2]. Pharyngeal narrowing or collapse leads to reduction or cessation in airflow during sleep, and is associated with loud snoring. In adults, risk factors include sedatives (alcohol, sleeping tablets), obesity, \& nasal obstruction. Sedatives, including alcohol, act by reducing muscle tone in the upper airway and pharynx, rendering them more likely to narrow and collapse [3].

The presenting symptom of OSA is usually excessive

\footnotetext{
*Trial registration. Clinical Trials. Gov NCT01322334.
}

daytime sleepiness. Patients often report a gradual deterioration of symptoms of snoring over several years to the point of presentation. A more rapid onset of severe symptoms should prompt enquiry towards metabolic abnormality predisposing to rapid weight gain or hypothyroidism, or the presence of pharyngeal pathology, e.g. tongue base lymphoma. Several studies have now shown a correlation of sleep apnoea with cardiovascular disease $[4,5]$. Moderate to severe sleep apnoea, as defined by Apnoea-Hypopnea Index (AHI) greater than 20, is associated with an excess risk of hypertension and mortality from stroke and ischaemic heart disease. The Wisconsin Sleep Cohort study [6] also identified an association of increased motor vehicle accidents with OSA. Although these factors are reliable correlates, it is not clear whether OSA is the cause of the problems; indeed there are considerable confounding factors of, for example, obesity, diabetes. 
Conventional definition of OSA has been on the basis of an AHI value: a greater value implying progressively more severe disease. However, there is generally poor correlation between sleep study parameters and self report of sleepiness. Many would now consider that severe symptoms of excessive daytime somnolence carry equal weight in determining pragmatic approach to treatment [7].

High quality evidence for treatments associated with snoring and sleep apnoea is limited. Conservative measures, such as weight reduction, treating nasal obstruction, reduction of evening alcohol and hypnotics are widely recommended and seem sensible and reasonable approach for many people but have no evidence base. [3] Continuous positive airways pressure (CPAP) is the main treatment modality for OSA in adults [8].

CPAP therapy in patients with moderate to severe sleep apnoea is associated with a significant improvement in daytime sleepiness and overall quality of life [9]. There is a reduction in blood pressure when OSA patients are treated with CPAP, but no evidence that intervention with CPAP or any other modality of therapy alters long term morbidity and mortality from cardiovascular disease $[10,11]$.

Tonsillectomy and adenoidectomy is a successful surgical treatment for many children suffering with OSA due to tonsil and adenoidal hypertrophy. Evidence for other treatments; uvulopalatopharyngoplasty, lingualplasty, mandibulotomy and advancement, hyoid suspension is largely based on case series or non-randomised trials. Success rates for surgery are probably no better than $50 \%$ in the long term, and can be notoriously painful [12].

A local singing teacher (AO) observed that some patients undergoing formal singing training, which involved exercises of repetitive contraction-relaxation cycles of pharyngeal muscles over a period of several weeks, reported reduced snoring and improved sleep as a consequence.

We hypothesised that regular exercise of this nature could strengthen pharyngeal muscles and/or increase their resting tone, and lead to an improvement of symptoms and thus quality of life in patients with all forms of snoring, including OSA.

\section{Methods}

\subsection{Population}

All patients were recruited to the study through the Otolaryngology department of the Royal Devon \& Exeter NHS Foundation Trust. Participation in the study was offered amongst a range of other treatment considerations to all patients seen in routine otolaryngology clinics with snoring or suspected sleep apnoea. Patients who expressed an interest returned for further assessment and trial information to a dedicated research clinic. Eligible patients were aged 18 or over, with a history of simple snoring or obstructive sleep apnoea with an apnoea index (AI) of 10 - 40. Exclusion criteria included severe sleep apnoea (AI > 40), severe obesity (BMI > 40) and concurrent use of CPAP therapy. Patients willing to participate gave their written informed consent.

The study was given approval by the North and East Devon Local Research Ethics committee.

\subsection{Randomization}

When patients had entered the trial and given their written consent, baseline measurements were taken (see below: "outcome measures"). Patients were then randomised to experimental or control groups, by way of sealed, opaque envelope allocation. Patients were stratified by diagnosis and a BMI cutoff to equally distribute the proportion of those with SS versus OSA, and BMI of $<30$ versus $30-40$ between the groups.

\subsection{Intervention and Control}

General advice was given to all patients about the importance of optimizing body weight, and reducing evening alcohol and sedatives. In the intervention group, the patients were given a triple CD set containing the singing exercises ("Singing for Snorers", UK). The programme is a self-guided treatment, with recommendation to spend minimum of 20 minutes per day on the exercises, and patients were instructed to follow the exercises as detailed in the CD's for a study period of 3 months. After a period of 4 - 6 weeks the singing teacher (AO) who devised the exercises made 1 phone call to the patient, to offer support and answer any technical questions about the exercises. In the control group, the patients had no intervention, but received a similarly timed phone call from a researcher enquiring about their progress. Patients in the control group were offered the triple CD set of exercises (at no charge) after the 3 month study period.

\subsection{Outcome Measures}

Our primary outcome measure was the Epworth sleepiness scale, a widely used, validated and reliable measure of sleep quality, and is rated from 0 to 24; higher scores representing greater daytime somnolence $[13,14]$. Secondary outcome measures included visual analogue scales (VAS: $0-10 \mathrm{~cm}$ ) as rated by the patient and/or partner of snoring loudness and frequency, and the SF-36 generic quality of life assessment tool [15]. Patients in the experimental group were asked about their compliance with the exercises, on a VAS of $0-10 \mathrm{~cm}$ representing "never doing the exercises" to "doing the exercises every day". 
Baseline values for all measures were taken at the point of entry to the trial before randomization. Patients returned for review after 3 months, and were instructed to complete the outcome rating forms (Epworth, VAS, SF-36) by clinic staff who were blind to their treatment group. Follow up sleep studies were not performed in any patients. Changes in patients' weight, smoking and alcohol intake were noted.

Patients who did not attend their initial 3 month follow up appointment were sent a further appointment; then phoned with a further appointment date; and finally if they still failed to attend their follow up appointment, the outcome measure sheets were posted to them.

\subsection{Analysis \& Power Calculation}

It was assumed that the control group would not show any significant improvement in symptoms over the course of the 3 month study period. There is no "normal" value for an Epworth score or the VAS's, the power of the study was arbitrarily set to detect a difference between groups of $20 \%$.

This calculation provides a sample size of 24. Estimating that $20 \%$ of patients in the experimental group may not comply well with the exercises, sample size for each group is set at 30 (assuming $\alpha=0.05,1-\beta=80 \%$ ).

Data was analysed on an intention to treat basis. Independent variables were considered as baselines values of outcome measures, group allocation, and snoring status (SS vs OSA). Dependent variables were considered to be outcome data recorded after 3 months, and t-tests were used to analyse the change scores or primary and secondary outcome measures (i.e. difference between scores at baseline and follow up). Where data were not normally distributed, they were summarised using medians and interquartile ranges. Comparisons were then made using nonparametric tests such as the Mann Whitney U test.

Analysis of covariance was performed looking particularly at the effect of snoring status (SS vs OSA) and BMI.

\section{Results}

Figure 1 is a CONSORT diagram [16] showing the flow of participants through the study. 127 patients were entered into the study ( 72 with SS, 55 with OSA). There was a high rate of loss to follow up, with complete data being available on only 93 patients. Average age, smoking, alcohol intake, and weight change did not different significantly between the groups. The sex ratio of groups was not uniformly distributed (Table 1).

Patients in the experimental group rated their compliance with exercises as a median of 6.6 (quartiles $=4.1$, 8.3). 3 patients did not perform the exercises at all. $65 \%$ of patients rated their compliance with the exercises as

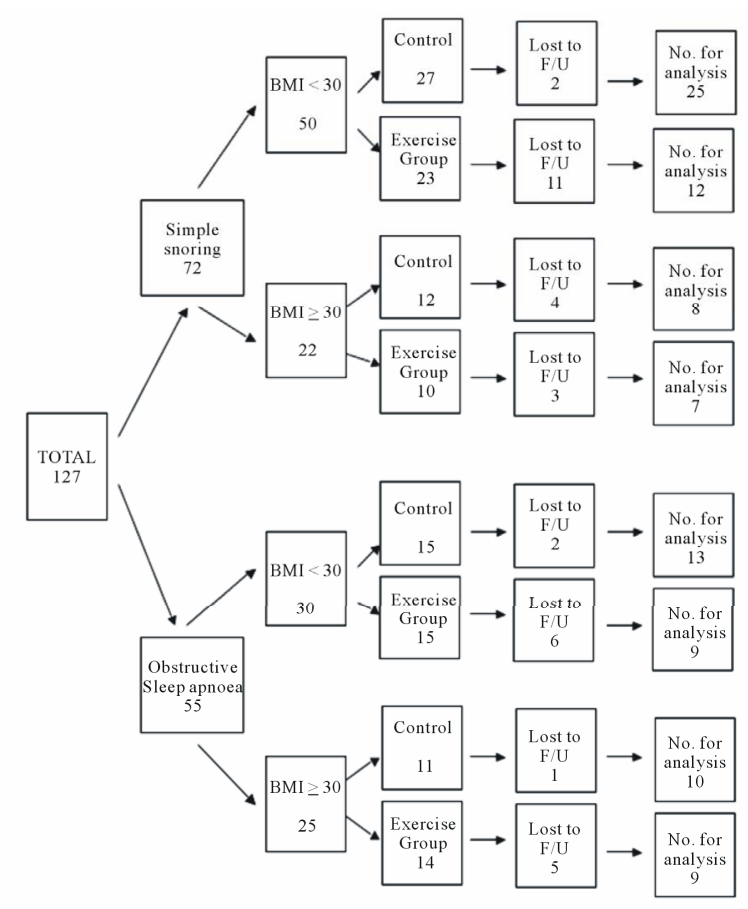

Figure 1. Group allocation and numbers of patients for analysis.

greater than 5 on the VAS. The Epworth scale improved significantly in the experimental group compared to the control group (difference -2.5 units; $95 \%$ CI -3.8 to -1.1 ; $\mathrm{p}=0.000$ ). Frequency of snoring reduced significantly in the experimental group (difference $-1.5 ; 95 \% \mathrm{CI}-2.6$ to $-0.4 ; \mathrm{p}=0.01)$, and loudness of snoring showed a trend to improvement which was non-significant (difference $-0.8 ; 95 \% \mathrm{CI}-1.7$ to $0.1 ; \mathrm{p}=0.08)$.

Scores on the SF-36 were low in the whole study population pre-intervention in the domains of health, vitality and mental health. There was a slight, but nonsignificant improvement in median scores in the experimental group after intervention.

When considering estimated marginal means of change of dependent co-variables, in a unilateral analysis of variance, improvement in Epworth scores showed a trend to greater effect amongst patients with OSA and/or a BMI of $>30$. Improvement in snoring loudness and frequency was greater for patients with $\mathrm{BMI}<30$. None of these results reached a level of statistical significance.

No adverse effects of treatment were recorded.

\section{Discussion}

This is a prospective, randomised, single blinded trial of which has demonstrated that an intervention with a self-guided instruction programme of specially tailored singing exercises, when performed over a 3 month period by patients with a range of severity of snoring problems, significantly improves daytime sleepiness, and snoring 
Table 1. Patient demographics and risk factors by group.

\begin{tabular}{|c|c|c|c|c|c|c|}
\hline Group & $\begin{array}{l}\text { Sex } \\
\text { F/M }\end{array}$ & Mean Age & $\begin{array}{c}\text { Smokers } \\
\text { (\% of group who } \\
\text { smoked daily) }\end{array}$ & $\begin{array}{l}\text { Mean pre-trial } \\
\text { weight } \\
(\mathrm{kg})\end{array}$ & $\begin{array}{l}\text { Mean post-trial } \\
\text { weight }\end{array}$ & $\begin{array}{l}\text { Mean daily } \\
\text { alcohol intake } \\
\text { (units) }\end{array}$ \\
\hline $\begin{array}{c}\text { SS } \\
\text { BMI }<30 \\
\text { Control }\end{array}$ & $8 / 16$ & 59 & $4 \%$ & 75.7 & 75.6 & 0.8 \\
\hline $\begin{array}{c}\text { SS } \\
\text { BMI }<30 \\
\text { Exercises }\end{array}$ & $9 / 3$ & 59 & $17 \%$ & 72.4 & 72.6 & 0.8 \\
\hline $\begin{array}{c}\text { SS } \\
\text { BMI } 30-40 \\
\text { Control }\end{array}$ & $5 / 3$ & 55 & $13 \%$ & 87.2 & 87.5 & 0.9 \\
\hline $\begin{array}{c}\text { SS } \\
\text { BMI } 30-40 \\
\text { Exercises }\end{array}$ & $3 / 4$ & 56 & $14 \%$ & 95.9 & 94.0 & 1.3 \\
\hline $\begin{array}{c}\text { OSA } \\
\text { BMI }<30 \\
\text { Control }\end{array}$ & $5 / 8$ & 59 & $8 \%$ & 77.2 & 77.3 & 0.8 \\
\hline $\begin{array}{c}\text { OSA } \\
\text { BMI }<30 \\
\text { Exercises }\end{array}$ & $3 / 6$ & 60 & $11 \%$ & 79.6 & 82.3 & 0.7 \\
\hline $\begin{array}{c}\text { OSA } \\
\text { BMI } 30-40\end{array}$ & $4 / 6$ & 52 & $10 \%$ & 96.9 & 95.6 & 0.7 \\
\hline $\begin{array}{c}\text { OSA } \\
\text { BMI } 30-40\end{array}$ & $3 / 6$ & 54 & $11 \%$ & 95.3 & 93.9 & 0.2 \\
\hline
\end{tabular}

frequency.

It is hypothesised that the singing exercises act by im proving the tone and strength of pharyngeal muscles, thereby reducing their tendency to collapse during sleep: one of prime factors in snoring and sleep apnoea. Similar improvement was noted by Puhan et al. [17] who found that didgeridoo playing for 4 months resulted in significantly reduced daytime somnolence, improvements in sleep quality, and improvements in apnoea-hypopnea index.

Several other treatments for primary snoring and obstructive sleep apnoea should be considered. In patients with sleep apnoea, CPAP therapy remains the gold standard for treatment with a strong evidence base for effective treatment of excessive daytime somnolence and quality of life [9]. Oral appliances, such as mandibular advancement devices are also effective for some patients which although less effective than CPAP [18]. A number of surgical interventions for sleep apnoea have been proposed: uvulopalatopharyngoplasty, laser assisted uvulopalatoplasty, mandibular advancement osteotomies and hyoid advancement. Evidence for their efficacy from randomised controlled trials against control or CPAP therapy is lacking [19].

Nasal appliances and nasal airway surgery is often recommended for primary snoring. There is no evidence of effect for nasal surgery in improving symptoms of snoring, although there is positive benefit in some OSA patients who show better tolerance to CPAP therapy after correction of nasal airway abnormalities [20]

The principal surgical intervention for primary snoring is laser uvulopalatoplasty, radiofrequency palatal ablation, or palatal implants. These techniques demonstrate improvement of symptoms in the short term [21,22]. Longer term results for palatal surgery diminish over time, with only $34 \%$ of patients reporting good symptom control after 2 years [23]. Although the other techniques of ablation and implant are less painful and associated with fewer initial complications, data on their long term efficacy is lacking.

There are no trials which assess the treatment effect of pharyngeal exercises against other modalities of snoring and sleep apnoea intervention, either medical or surgical. Given the heterogeneity of patient symptoms and assessment across trials, it is not possible to make a statement of efficacy of pharyngeal treatments in comparison to other possible interventions.

Strengths and Limitations of the Trial

This is a large prospective randomised controlled trial which applies a well defined intervention for a 3-month period, to a diverse group of patients who presented to a general otolaryngology clinic with primary complaint of snoring and/or sleeping problems. Appropriate block randomisation has ensured an even distribution of important co-factors (sleep apnoea status, BMI) between the control and intervention group. The intervention was well tolerated, and there was generally good compliance amongst patients with the exercises, with no adverse 
effects.

There are a number of limitations of the study. The high loss to follow up (data was available for $73.2 \%$ of participants) is a cause for concern. This was despite robust attempts to contact patients with follow up appointments, phone calls, and contact by post. This loss to follow up makes it difficult to provide any realistic estimate of what proportion of patients might expect to benefit from the intervention.

The outcome measures did not include a repeat sleep study in the subgroup of patients who entered the trial with a diagnosis of sleep apnoea (AHI 10-40). The Epworth scale is a validated measure of daytime somnolence, but the degree of confidence in the effect of intervention would be further enhanced with post-intervention sleep study demonstrating a clinically and statistically significant improvement in the $\mathrm{AI}$ in the experimental group. This was not available because of lack of funding.

Patients were not blinded to the intervention, and there was no comparable activity in the control group. It was felt that any attempt to perform throat exercises or movements might produce a specific, rather than placebo effect. Assessors were blind to the status of the patient. The principal outcome measure is the Epworth score. Although reliable, it remains a subjective measure and might be subject to bias from patients who were not blinded to treatment group.

In conclusion, this study adds weight to the hypothesis that improving the tone and strength of pharyngeal muscles reduces the severity, frequency and loudness of snoring, and improves mild to moderate sleep apnoea. The intervention has been applied to a study population which accurately reflects a cross section of patients presenting to secondary care in the UK with difficulties due to snoring, and has no adverse effects. In the context of other treatments which are more invasive, and may be poorly tolerated and painful, it should be considered as an alternative modality of treatment. Further studies in specific patient groups are needed to define the magnitude of effect.

\section{Data Sharing}

No additional data available.

All authors declare: No support from any organization for the submitted work; no financial relationships with any organizations that might have an interest in the submitted work in the previous 3 years; no other relationships or activities that could appear to have influenced the submitted work.

\section{Study Sponsor}

The CD's of the singing exercises were provided free for the purpose of the study by the developer (AO) "Singing for Snorers, UK". The researchers are independent from the sponsor. The sponsor played no role in study design, data collection, data analysis, data interpretation, report writing, or decision to submit for publication.

\section{REFERENCES}

[1] J. R. Stradling, "Obstructive Sleep Apnoea: Definitions, Epidemiology, and Natural History," Thorax, Vol. 50, 1995, pp. 683-689. doi:10.1136/thx.50.6.683

[2] M. I. Trotter and D. W. Morgan, "Snoring: Referral, Investigation and Treatment," British Journal of Hospital Medicine (London), Vol. 68, No. 2, 2005, pp. 95-99.

[3] P. Counter and J. A. Wilson, "The Management of Simple Snoring," Sleep Medicine Reviews, Vol. 8, No. 6, 2004, pp. 433-441. doi:10.1016/j.smrv.2004.03.007

[4] P. Lavie, "Sleep Apnea Causes Cardiovascular Disease," American Journal of Respiratory and Critical Care Medicine, Vol. 169, No. 2, 2004, pp. 147-148. doi: $10.1164 / \mathrm{rccm} .2310010$

[5] J. Wright, R. Johns, I. Watt, et al., "Health Effects of Obstructive Sleep Apnoea and the Effectiveness of Continuous Positive Airways Pressure: A Systematic Review of the Research Evidence," British Medical Journal, Vol. 314, No. 7084, 1997, pp. 851-860. doi:10.1136/bmj.314.7084.851

[6] K. M. Rex, D. F. Kripke and M. R. Klauber, "Sleep-Disordered Breathing in Middle-Aged Adults Predicts Significantly Higher Rates of Traffic Violations," Chest, Vol. 119 , No. 5, 2001, pp. 1623-1624. doi: $10.1378 /$ chest.119.5.1623

[7] J. R. Stradling and R. J. O. Davies, “Obstructive Sleep Apnoea/Hypopnoea Syndrome: Definitions, Epidemiology, and Natural History," Thorax, Vol. 59, No. 1, 2004, pp. 73-78. doi:10.1136/thx.2003.007161

[8] N. Freedman, "Treatment of Obstructive Sleep Apnea Syndrome," Clinics in Chest Medicine, Vol. 31, No. 2, 2010, pp. 187-201. doi:10.1016/j.ccm.2010.02.012

[9] T. L. Giles, T. J. Lasserson, B. Smith, J. White, J. J. Wright, C. J. Cates, "Continuous Positive Airways Pressure for Obstructive Sleep Apnoea in Adults," Cochrane Database of Systematic Reviews, No. 3. 2006, Article ID: CD001106.

[10] J. C. T. Pepperell, S. Ramdassing-Dow, N. Crosthwaite, et al., "Ambulatory Blood Pressure Following Therapeutic and Sub-Therapeutic Nasal Continuous Positive Airway Pressure for Obstructive Sleep Apnoea: A Randomised Prospective Parallel Trial," Lancet, Vol. 359, No. , 2002, pp. 204-210. doi:10.1016/S0140-6736(02)07445-7

[11] J. F. Faccenda, T. W. Mackay, N. A. Boon, et al., "Randomized Placebo-Controlled Trial of Continuous Positive Airway Pressure on Blood Pressure in the Sleep ApneaHypopnea Syndrome," American Journal of Respiratory and Critical Care Medicine, Vol. 163, No. 2, 2001, pp. 344-348.

[12] A. E. Sher, K. B. Schechtman and J. F. Piccirillo, "The Efficacy of Surgical Modifications of the Upper Airway in Adults with Obstructive Sleep Apnea Syndrome," 
Sleep, Vol. 19, No. 2, 1996, pp. 156-177.

[13] M. W. Johns, "A New Method for Measuring Daytime Sleepiness: The Epworth Sleepiness Scale," Sleep, Vol. 14, No. 6, 1991, pp. 540-545.

[14] M. W. Johns, "Reliability and Factor Analysis of the Epworth Sleepiness Scale," Sleep, Vol. 15, No. 4, 1992, pp. 376- 381. PMid:1519015

[15] A. M. Garratt, D. A. Ruta, M. I. Abdalla, J. K. Buckingham and I. T. Russell, "The SF36 Health Survey Questionnaire: An Outcome Measure Suitable for Routine Uses within the NHS?" British Medical Journal, Vol. 306, No. 6890, 1993, pp. 1440-1444. doi:10.1136/bmj.306.6890.1440

[16] K. F. Schulz, D. G. Altman and D. Moher for the CONSORT Group, "CONSORT 2010 Statement: Updated guidelines for reporting parallel group randomised trials," British Medical Journal, Vol. 340, 2010, p. c332. doi:10.1136/bmj.c332

[17] M. A. Puhan, A. Suarez, C. Lo Cascio, A. Zahn, M. Heitz and O. Braendli, "Didgeridoo Playing as Alternative Treatment for Obstructive Sleep Apnoea Syndrome: Randomised Controlled Trial," British Medical Journal, Vol. 332, No. 7536, 2006, pp. 266-270. doi:10.1136/bmj.38705.470590.55

[18] J. Lim, T. J. Lasserson and J. Wright, "Oral Appliances for Obstructive Sleep Apnoea," Cochrane Database of
Systematic Reviews, No. 4, 2006, Article ID: CD004435.

[19] S. Sundaram, S. A. Bridgman, J. Lim and T. J. Lasserson, "Surgery for Obstructive Sleep Apnoea," Cochrane Database of Systematic Reviews, No. 4, 2005, Article ID: CD001004.

[20] M. Friedman, H. Tanyeri, J. W. Lim, R. Landsberg, K. Vaidyanathan and D. Caldarelli, "Effect of Nasal Breathing on Obstructive Sleep Apnea," Otolaryngology-Head and Neck Surgery, Vol. 122, No. 1, 2000, pp. 71-74. doi:10.1016/S0194-5998(00)70147-1

[21] J. T. Maurer, T. Verse, B. A. Stuck, K. Hormann and G. Hein, "Palatal Implants for Primary Snoring: Short-Term Results of a New Minimally Invasive Surgical Technique," Otolaryngology_Head and Neck Surgery, Vol. 132, No. 1, 2005, pp. 125-131. doi:10.1016/j.otohns.2004.09.015

[22] L. J. Bäck, M. L. Hytönen, R. P. Roine and A. O. Malmivaara, "Radiofrequency Ablation Treatment of Soft Palate for Patients with Snoring: A Systematic Review of Effectiveness and Adverse Effects," Laryngoscope, Vol. 119, No. 6, 2009, pp. 1241-1250. doi:10.1002/lary.20215

[23] T. M. Jones, J. E. Earis, P. M. Calverley, S. De and A. C. Swift, "Snoring Surgery: A Retrospective Review," Laryngoscope, Vol. 115, No. 11, 2005, pp. 201-205. doi:10.1097/01.mlg.0000180178.12972.81 